\title{
Percepción parental de la salud psicofísica, estado nutricional y salud bucal, en relación con características sociodemográficas en niños de Bariloche, Argentina: estudio epidemiológico
}

\author{
Parental perception of psychophysical health, nutritional \\ status and oral health in relation to sociodemographic \\ characteristics in children in Bariloche, Argentina: \\ an epidemiological study
}

Dra. en Estadística Gilda Garibottia , Lic. en Trabajo Social Cecilia Vasconi ${ }^{b}$, Dra. Alejandra Ferrari, ${ }^{\circledR}$ Dra. Gabriela Giannini ${ }^{d}$ Dra. Haydeé Comarc,dy Dr. Diego Schnaiderman ${ }^{d}$

a. Departamento de Estadística, Centro Regional Universitario Bariloche, Universidad Nacional del Comahue, Bariloche, Río Negro, Argentina.

b. Secretaría de Salud. Municipalidad de Bahía Blanca, Buenos Aires, Argentina.

c. Centro Regional Universitario Bariloche, Universidad Nacional del Comahue.

d. Consultorios pediátricos privados. Bariloche, Río Negro, Argentina.

Correspondencia: Dra. Gilda Garibotti: garibottig@comahueconicet.gob.ar

\section{Financiamiento:} Esta investigación fue parcialmente financiada por la Universidad Nacional del Comahue. Subsidio de la Secretaría de Investigación B188.

Conflicto de intereses: Ninguno que declarar.

Recibido: 16-10-2014 Aceptado: 13-4-2015

\section{RESUMEN}

Introducción. Existen evidencias de la asociación de determinantes sociales con la salud infantil. Objetivo. Identificar características sociodemográficas asociadas a desigualdades en la salud infantil y evaluar el efecto acumulado sobre la salud de factores de riesgo basados en estas características.

Población y métodos. Evaluamos niños de 4-13 años, de Bariloche, entre junio de 2008 y mayo de 2009. Características sociodemográficas consideradas: nivel socioeconómico, educación materna, embarazo adolescente, cobertura médica, inseguridad y hábitos familiares. Valoramos la percepción parental de la salud física y socioemocional, el estado nutricional y la salud bucal en relación con dichas características y con la acumulación de factores de riesgo. Utilizamos encuesta, antropometría y examen bucal.

Resultados. Participaron 180 escolares. El nivel educativo materno se asoció con la salud física, socioemocional y bucal del niño. El porcentaje de niños con piezas faltantes o caries fue $77 \%$ entre aquellos cuyas madres, como máximo, habían completado el primario, comparado con $13 \%$ entre aquellos cuyas madres habían completado estudios terciarios/universitarios.

La posibilidad de percepción de salud física y socioemocional no óptima aumentó con cada factor de riesgo 1,8 y 1,4 veces, respectivamente, y la posibilidad de caries o piezas faltantes se duplicó con cada factor de riesgo adicional.

El 27,3\% de los escolares presentó sobrepeso y el 8,7\%, obesidad, y no se encontró asociación con características sociodemográficas.

Conclusiones. El bajo nivel socioeconómico familiar y educativo materno se asoció con una mayor prevalencia de resultados de salud desfavorables. Múltiples factores de riesgo tienen un efecto acumulado sobre la percepción parental de la salud física y socioemocional y la salud bucal.

Palabras clave: desigualdades en la salud, epidemiología, salud del niño, Argentina. http://dx.doi.org/10.5546/aap.2015.411

\section{INTRODUCCIÓN}

La salud infantil mejoró notablemente durante el siglo XX debido, entre otros factores, a la reducción de la ocurrencia de enfermedades infecciosas y la mortalidad general. ${ }^{1}$ Sin embargo, estas mejoras no ocurrieron con igual intensidad en todos los países ni en todas las regiones dentro de cada país. ${ }^{2}$

Numerosas investigaciones encontraron disparidades en diversos aspectos de la salud en la población pediátrica argentina. La asociación entre mayor nivel socioeconómico (NSE) y mejor desempeño en pautas de desarrollo infantil está bien establecida, incluso en estudios realizados en Argentina..$^{3-5}$ Argentina, al igual que otros países en vías de desarrollo, está experimentando un importante incremento en la prevalencia de sobrepeso y obesidad infantil. Los resultados en cuanto a la asociación entre NSE y sobrepeso y obesidad son dispares. Si bien, décadas atrás, la obesidad se consideraba un problema de los sectores sociales altos, actualmente, el problema afecta a niños de distintos sectores sociales en similar medida. ${ }^{6-10}$ En distintas regiones del país, se determinó que los niños pertenecientes a familias con menores 
ingresos presentaban mayor nivel de enfermedad bucal. ${ }^{11,12}$ Las tasas de mortalidad infantil varían dependiendo del NSE de la población; son más altas en los grupos con mayor prevalencia de necesidades básicas insatisfechas. ${ }^{13,14}$

Los estudios epidemiológicos sobre indicadores de salud en la niñez asociados con las condiciones de vida permiten conocer la magnitud y distribución de desigualdades. Este conocimiento es necesario al momento de diseñar políticas de salud tendientes a reducir las inequidades. Actualmente, la salud se define no solo como la ausencia de enfermedad, sino como un estado de completo bienestar psicofísico y social que les permite desarrollar y alcanzar sus potencialidades, satisfacer sus necesidades y adquirir capacidades para interactuar exitosamente con su entorno. ${ }^{15,16}$ Desde esta perspectiva, evaluar la salud infantil requiere considerar un espectro amplio de indicadores.

El presente trabajo tiene como objetivo identificar características sociodemográficas asociadas a desigualdades en la percepción parental de la salud física y socioemocional del niño, el estado nutricional y la salud bucal, y evaluar el efecto acumulado de factores de riesgo asociados a estas características sobre la salud en la población en edad escolar de la ciudad de Bariloche.

\section{POBLACIÓN Y MÉTODOS}

El estudio se llevó a cabo sobre la base de una encuesta anónima realizada al padre o la madre de niños de 4 a 13 años residentes en Bariloche y del relevamiento antropométrico y de salud bucal de los niños (Anexo 1). Las encuestas se realizaron en el Centro de Salud del barrio 2 de Abril (uno de los barrios de menor NSE de la ciudad), en consultorios de las obras sociales de Trabajadores Gastronómicos y Empleados de Comercio y en un centro pediátrico privado. Se presume que las familias atendidas en estos centros representan los distintos sectores sociales de la ciudad. Se excluyeron niños con enfermedades crónicas con posible efecto sobre su desarrollo psicofísico.

En ausencia, a nivel local, de datos previos sobre la distribución de las medidas de salud de interés, se estableció un tamaño muestral de 200 niños, aproximadamente $1 \%$ de la población de 5 a 14 años de Bariloche (Censo 2001, INDEC). En los consultorios de las obras sociales y del centro privado, los participantes se seleccionaron aleatoriamente entre los pacientes con turno de los médicos pediatras coautores de este estudio: un paciente por día por pediatra. En el Centro de Salud, las encuestas fueron realizadas por uno de los médicos pediatras en diversos días y horarios. Las entrevistas se realizaron entre junio de 2008 y mayo de 2009. Se invitó a participar a las madres y a los padres; se les brindó información sobre el proyecto y se les solicitó su consentimiento verbal. El protocolo de investigación fue aprobado por el Comité de Docencia e Investigación del Hospital Zonal Bariloche.

La encuesta fue elaborada utilizando como modelo la Encuesta Nacional sobre Salud Infantil 2003 de Estados Unidos (2003 National Survey of Children's Health). ${ }^{17,18}$ Esta fue tomada como referencia por la variedad de aspectos de la salud que aborda y por recabar la percepción parental sobre la salud de los niños.

\section{Medidas de salud de los niños}

Las medidas de salud consideradas fueron percepción parental del estado de salud física y socioemocional del niño, estado nutricional y salud bucal.

- Salud física. De acuerdo con la percepción parental, el estado de salud del niño fue clasificado por el encuestado en excelente, muy bueno, bueno, regular o pobre. Para los análisis, se dividió en óptimo (muy bueno/ excelente) y no óptimo (pobre/regular/ bueno).

- Salud socioemocional. Se preguntó al padre/ madre si estaba preocupado o no respecto del comportamiento del niño, desenvolvimiento escolar, capacidad de entender consignas, relación con otros niños y con adultos (maestros, padres, etc.), depresión o ansiedad, capacidad para resolver situaciones de conflicto y forma de hablar. Se calculó el número total de tópicos sobre los que el encuestado estaba preocupado. La distribución de las respuestas se dividió en cuartiles; se consideraron con salud socioemocional no óptima los niños del cuartil superior. La preocupación parental tiene alto valor predictivo sobre la salud socioemocional. ${ }^{19,20}$

- Estado nutricional. Se registró el peso de los niños utilizando balanzas de pie tipo CAM, sus altímetros para medir tallas mayores de $110 \mathrm{~cm}$ y pediómetros para tallas menores de $110 \mathrm{~cm}$. Sobre la base del índice de masa corporal (IMC), los niños fueron categorizados en eutróficos (peso normal), con sobrepeso, obesos y delgados utilizando la referencia internacional de Obesity Task Force. ${ }^{21,22}$ Para los 
análisis, se dividió en sobrepeso u obesidad (sí/no).

- Salud bucal. El médico verificó la presencia de caries y la falta de piezas dentarias, resultante de la extracción intencional por un odontólogo a causa de enfermedad bucal (sí/no).

\section{Características sociodemográficas}

Las características sociodemográficas consideradas fueron nivel de bienestar económico familiar (NBEF), nivel educativo máximo alcanzado por la madre, embarazo adolescente, tipo de cobertura médica, barrio o escuela inseguro, hogar uniparental, hábito de lectura y tiempo frente al televisor y/o computadora.

- Nivel de bienestar económico familiar. Se empleó un modelo similar al propuesto por Currie y cols. ${ }^{23}$ que consiste en considerar un conjunto de artículos que reflejan el nivel de gastos familiares. Se registró el número de respuestas afirmativas a los siguientes puntos: la familia posee auto, posee computadora, no recibe subsidios, el niño salió de vacaciones, al menos, una vez durante el último año y el número de personas con las que el niño comparte el dormitorio es menor de 3. Los valores mayores se asocian a un nivel de bienestar económico familiar más alto. Categorías: bajo (0 o 1); medio (2, 3 o 4); alto (5).

- Nivel educativo máximo alcanzado por la madre. Se consideraron las categorías: ninguno/primario, secundario, terciario/ universitario.

- Embarazo adolescente. Refiere a embarazo de menores de 19 años.

- Tipo de cobertura médica. Se consideraron las categorías ninguna, obra social, prepaga.

- Barrio o escuela inseguros. Percepción parental de la inseguridad del niño en el barrio o escuela. Fue clasificado por el encuestado en nunca, a veces, siempre o usualmente. Para el análisis, se dividió en nunca/a veces, contra siempre/usualmente.

- Hogar uniparental. Hogar compuesto por solo uno de los progenitores.

- Hábito de lectura. El niño que había leído o al que le habían leído, al menos, una vez un cuento o parte de una novela durante la última semana se consideró que tenía hábito de lectura.

- Tiempo frente al televisor. Se distinguió a los niños expuestos a la computadora o la televisión 3 o más horas diarias de los expuestos menos de 3 horas.
Se consideraron factores de riesgo: NBEF bajo, nivel educativo máximo materno ninguno/ primario, embarazo adolescente, no contar con cobertura médica, inseguridad en el barrio o la escuela, hogar uniparental, no tener hábito de lectura y 3 o más horas diarias expuesto al televisor o a la computadora.

\section{Análisis estadístico}

Se determinó la media y el desvío estándar de la edad de los niños estudiados y la distribución porcentual de las medidas de salud y de las características sociodemográficas consideradas.

Para cada medida de salud, se estudió la relación de las características sociodemográficas de manera individual, con la posibilidad de presentar un resultado desfavorable para dicha medida, utilizando la prueba chi-cuadrado de Pearson.

La asociación entre el número de factores de riesgo y las medidas de salud se examinó utilizando el modelo de regresión logística.

El procesamiento y análisis de la información se realizó utilizando el paquete estadístico $R$ 3.1.1. ${ }^{24}$

\section{RESULTADOS}

Participaron 180 niños: 53 del Centro de Salud del barrio 2 de Abril, 61 de consultorios de las obras sociales de Trabajadores Gastronómicos y Empleados de Comercio, y 66 del centro pediátrico privado. Ninguna familia rehusó ser entrevistada. La media de la edad fue 8,1 años (desvío estándar: 2,8 años), y el 51\% fueron varones. En el $80 \%$ de los casos, fue entrevistada la madre. Las estadísticas descriptivas de las medidas de salud, las características sociodemográficas y el número de factores de riesgo se muestran en la Tabla 1. El $36 \%$ de los padres perciben que sus niños tienen salud física no óptima y el $23 \%$, que tienen salud socioemocional no óptima. El 36\% de los niños presentan sobrepeso u obesidad y casi el $50 \%$ tiene caries o piezas dentarias faltantes. La mayoría de los niños (83\%) presentaron algún factor de riesgo; aproximadamente, 50\% tenían 2 o más; y $28 \%$, cuatro o más factores de riesgo.

La Tabla 2 indica que existe un gradiente en la probabilidad de los niños de tener salud física no óptima en relación con el NBEF, que pasa de $9 \%$ en el NBEF alto a $79 \%$ en el NBEF bajo. Un patrón similar se observa en relación con la salud socioemocional y la salud bucal, pero no respecto al sobrepeso $u$ obesidad $(p=0,134)$. La educación materna terciaria/universitaria se asocia con 
menores posibilidades de un estado de salud física no óptimo, tanto al comparar con niños cuyas madres completaron el secundario como con aquellas con educación ninguna-primario. La salud bucal infantil empeora en relación inversa con el nivel educativo materno, y pasa del $77 \%$ en niños cuyas madres, como máximo, completaron el nivel primario a $13 \%$ entre aquellos cuyas madres completaron estudios terciarios o universitarios. No se encontraron diferencias significativas en la prevalencia de sobrepeso $u$ obesidad respecto a los factores estudiados.

La Figura 1 muestra el efecto de la acumulación de factores de riesgo sobre la salud infantil. El porcentaje de niños con salud física no óptima entre quienes no presentaron factores de riesgo fue aproximadamente $10 \%$ y se incrementó paulatinamente hasta el $90 \%$ entre aquellos con 6 o más atributos de riesgo. Se ajustó el modelo de regresión logística tomando como variable dependiente la salud física no óptima $(s i ́=1 ;$ no $=0)$ y, como variable independiente, el número de factores de riesgo. La razón de posibilidades (odds ratio) estimada fue 1,8 ( $\mathrm{p}<$ 0,001 ). La posibilidad de percepción parental de salud física no óptima se incrementó 1,8 veces con cada factor de riesgo adicional.

Del grupo de niños con ausencia de atributos de riesgo, menos del 5\% presentaron salud socioemocional no óptima, en contraste con el $50 \%$ entre aquellos con 5 o 6 factores de riesgo. La percepción parental de salud socioemocional

TABLA 1. Medidas de salud, características sociodemográficas y número de factores de riesgo en escolares de Bariloche $(n=180)$

\begin{tabular}{|c|c|c|}
\hline Característica & Categorías & n (\%) \\
\hline \multicolumn{3}{|l|}{ Medidas de salud } \\
\hline \multirow[t]{5}{*}{ Salud física* } & Excelente & $46(25,3)$ \\
\hline & Muy buena & $69(38,2)$ \\
\hline & Buena & $61(33,7)$ \\
\hline & Regular & $4(2,2)$ \\
\hline & Pobre & $1(0,6)$ \\
\hline Salud socioemocional ${ }^{*}$ & No óptima & $41(22,8)$ \\
\hline \multirow[t]{4}{*}{ Estado nutricional } & Normal & $109(60,5)$ \\
\hline & Delgado & $6(3,5)$ \\
\hline & Sobrepeso & $49(27,3)$ \\
\hline & Obesidad & $16(8,7)$ \\
\hline Caries o piezas faltantes & Sí & $89(49,4)$ \\
\hline \multicolumn{3}{|l|}{ Características sociodemográficas } \\
\hline \multirow{3}{*}{ Nivel de bienestar económico familiar } & Bajo & $28(15,7)$ \\
\hline & Medio & $91(50,6)$ \\
\hline & Alto & $61(33,7)$ \\
\hline \multirow[t]{3}{*}{ Nivel educativo máximo alcanzado por la madre } & Ninguno o primario & $79(44,1)$ \\
\hline & Secundario & $53(29,6)$ \\
\hline & Terciario o universitario & $47(26,3)$ \\
\hline Embarazo adolescente & Sí & $47(26,2)$ \\
\hline \multirow[t]{3}{*}{ Tipo de cobertura médica } & Ninguna & $46(25,8)$ \\
\hline & Obra social & $91(50,6)$ \\
\hline & Prepaga & $42(23,6)$ \\
\hline Barrio o escuela inseguro & Sí & $76(42,2)$ \\
\hline Hogar uniparental & Sí & $55(30,7)$ \\
\hline Hábito de lectura & No & $88(48,9)$ \\
\hline Tiempo frente al televisor & 3 o más horas & $28(15,7)$ \\
\hline \multirow[t]{8}{*}{ Número de factores de riesgo } & 0 & $30(16,7)$ \\
\hline & 1 & $52(28,9)$ \\
\hline & 2 & $27(15,0)$ \\
\hline & 3 & $20(11,1)$ \\
\hline & 4 & $20(11,1)$ \\
\hline & 5 & $12(6,7)$ \\
\hline & 6 & $12(6,7)$ \\
\hline & $7 \mathrm{u} 8$ & $7(3,9)$ \\
\hline
\end{tabular}

\footnotetext{
* Percepción parental del estado de salud.
} 
no óptima aumentó $1,4(\mathrm{p}<0,001)$ veces con cada factor de riesgo adicional.

La razón de posibilidades del modelo de regresión logística con la variable respuesta sobrepeso u obesidad no fue significativa.

El número de factores de riesgo también explica la salud bucal. Cada factor de riesgo adicional duplicó $(\mathrm{p}<0,001)$ la posibilidad de caries o piezas faltantes.

\section{DISCUSIÓN}

Las desigualdades en la salud constituyen un problema a nivel mundial. ${ }^{2}$ La Organización Mundial de la Salud concibe la inequidad sanitaria como las "desigualdades innecesarias y evitables y, además, injustas". ${ }^{25}$ Así, en términos operacionales, la equidad en la salud consiste en minimizar las disparidades evitables junto con sus determinantes entre las personas que tienen diferentes niveles de privilegios o ventajas. ${ }^{26}$ El primer paso para abordar esta problemática es la identificación de las disparidades y los factores que las determinan. Este estudio identificó importantes brechas en la salud de la población infantil de Bariloche respecto de todos los aspectos de la salud evaluados, salvo el nutricional.

El nivel educativo materno mostró una asociación significativa con el estado de salud física, socioemocional y bucal, y aumentó la prevalencia de resultados de salud desfavorables en relación inversa con el nivel educativo. El nivel educativo materno se vincula con pautas de crianza: alimentación, capacidad de crear un entorno que fomente el aprendizaje y el conocimiento, y compromiso con medidas preventivas para el cuidado dentario, entre otros. Además, es un indicador del alfabetismo en la salud, relevante porque el bajo alfabetismo en la salud afecta la comunicación entre el personal de salud y el paciente, dificulta la comprensión de tratamientos e incide en su abandono. ${ }^{27}$ En este sentido, son importantes las políticas que promueven la continuación de los estudios de jóvenes y adultos y los programas de salud, como el de sanidad escolar (ProSanE), que propone

TABla 2. Distribución porcentual de la prevalencia de la condición de salud desfavorable en escolares de Bariloche en relación con características sociodemográficas y familiares $(n=180)$. Comparación mediante prueba Chi-cuadrado

\begin{tabular}{|c|c|c|c|c|}
\hline $\begin{array}{l}\text { Características } \\
\text { sociodemográficas }\end{array}$ & $\begin{array}{l}\text { Salud física:* } \\
\text { no óptima }\end{array}$ & $\begin{array}{c}\text { Salud socioemocional:* } \\
\text { no óptima }\end{array}$ & $\begin{array}{l}\text { Sobrepeso } \\
\text { u obesidad }\end{array}$ & $\begin{array}{c}\text { Caries o piezas } \\
\text { faltantes } \\
\end{array}$ \\
\hline Nivel de bienestar económico familiar & $\mathrm{p}<0,001$ & $\mathrm{p}<0,001$ & $\mathrm{p}=0,134$ & $\mathrm{p}<0,001$ \\
\hline Bajo & 78,6 & 53,6 & 30,4 & 82,1 \\
\hline Medio & 42,7 & 23,3 & 43,7 & 60,0 \\
\hline Alto & 8,5 & 6,7 & 28,3 & 18,3 \\
\hline Nivel educativo máximo alcanzado por la madre & $\mathrm{p}<0,001$ & $\mathrm{p}<0,001$ & $\mathrm{p}=0,053$ & $\mathrm{p}<0,001$ \\
\hline Ninguno o primario & 59,0 & 38,0 & 46,6 & 77,2 \\
\hline Secundario & 30,2 & 17,0 & 28,8 & 39,6 \\
\hline Terciario o universitario & 4,3 & 4,3 & 28,3 & 12,8 \\
\hline Embarazo adolescente & $p=0,003$ & $p=0,167$ & $\mathrm{p}=0,450$ & $\mathrm{p}=0,004$ \\
\hline Sí & 59,3 & 35,7 & 42,3 & 75,0 \\
\hline No & 25,3 & 20,3 & 31,6 & 40,5 \\
\hline Tipo de cobertura médica & $\mathrm{p}<0,001$ & $p=0,001$ & $p=0,179$ & $\mathrm{p}<0,001$ \\
\hline Ninguna & 60,0 & 41,3 & 39,0 & 78,3 \\
\hline Obra social & 37,1 & 18,9 & 40,9 & 51,1 \\
\hline Prepaga & 7,1 & 9,5 & 24,4 & 11,9 \\
\hline Barrio o escuela inseguros & $\mathrm{p}<0,001$ & $p=0,010$ & $p=0,114$ & $\mathrm{p}<0,001$ \\
\hline Sí & 56,0 & 32,9 & 43,7 & 72,4 \\
\hline No & 22,3 & 15,4 & 30,7 & 32,7 \\
\hline Hogar uniparental & $\mathrm{p}<0,001$ & $p=0,263$ & $p=0,805$ & $p=0,04$ \\
\hline Sí & 58,2 & 29,1 & 34,0 & 61,8 \\
\hline No & 27,0 & 20,2 & 37,3 & 44,4 \\
\hline Hábito de lectura & $p=0,246$ & $p=1$ & $\mathrm{p}=0,350$ & $p=0,007$ \\
\hline No & 41,4 & 22,7 & 40,2 & 60,2 \\
\hline Sí & 31,9 & 22,8 & 32,2 & 39,1 \\
\hline Tiempo frente al televisor & $\mathrm{p}=0,041$ & $\mathrm{p}=0,551$ & $\mathrm{p}=0,182$ & $\mathrm{p}<0,001$ \\
\hline Más de 3 horas & 55,6 & 28,6 & 50,0 & 25 \\
\hline Menos de 3 horas & 32,9 & 21,3 & 34,0 & 42,7 \\
\hline
\end{tabular}

* Percepción parental del estado de salud. 
exámenes médicos, incluidos los bucales, para todos los alumnos de primero y sexto grado. ${ }^{28}$

La presencia de caries es una de las enfermedades crónicas más comunes en la infancia. Numerosos estudios hallaron relación entre educación materna y salud bucal. ${ }^{11,12,29}$ El $77 \%$ de los niños cuyas madres habían completado, como máximo, la educación primaria presentaron caries o piezas faltantes, comparado con $40 \%$ entre aquellos cuyas madres habían terminado el secundario y $13 \%$ entre los de madres que habían completado estudios terciarios o universitarios. Estos resultados ponen de manifiesto la necesidad urgente de implementar programas de promoción y prevención de la salud bucal. Esta enfermedad tan común es prevenible mediante el lavado diario de los dientes y el control del consumo de golosinas. ${ }^{30}$
Las prevalencias de sobrepeso y obesidad obtenidas son elevadas: $27,3 \%$ y $8,7 \%$, respectivamente. Sin embargo, estas prevalencias son similares a las encontradas en otros relevamientos sobre nutrición infantil realizados en diversas regiones del país, en los que los porcentajes variaron entre $10,9 \%$ y $25,6 \%$ para sobrepeso y entre 3,8\% y 13,8\% para obesidad. ${ }^{7,8,31}$ En la región patagónica, si bien no hay estudios comparativos, el problema tiende a ser mayor. ${ }^{8}$ Al igual que en otros estudios llevados a cabo en la Argentina, no se encontró asociación entre el estado nutricional y las características sociodemográficas estudiadas. ${ }^{6,810}$ Esta situación puede ser resultado del consumo de alimentos hipercalóricos y la falta de actividad física. Considerando su alta prevalencia, la obesidad debe ser un tema prioritario de salud pública.

FIgura 1. Porcentaje de niños en edad escolar de Bariloche con resultado desfavorable en salud, en relación con el número de factores de riesgo
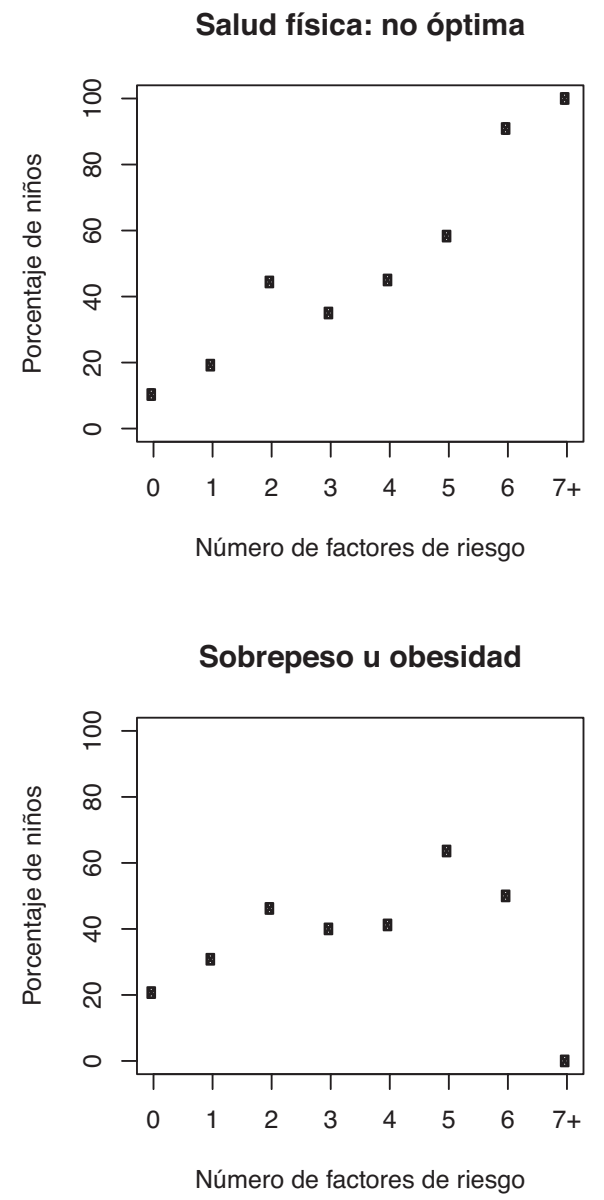

Salud socioemocional: no óptima

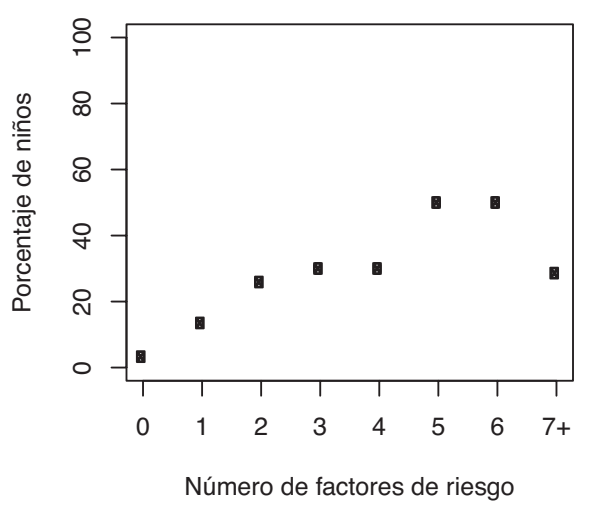

Presencia de caries o piezas faltantes

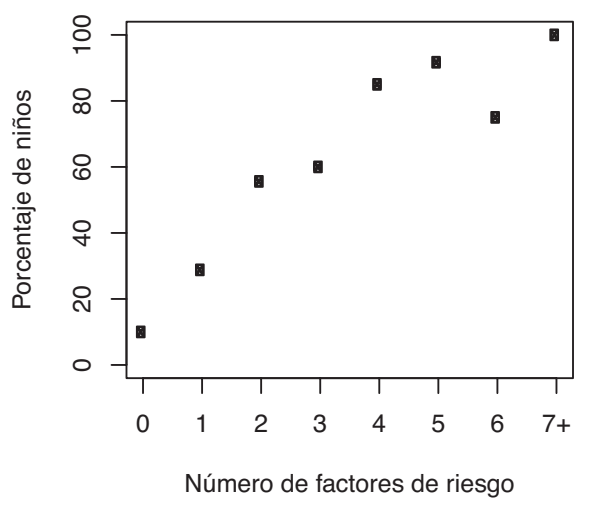


Los niños con sobrepeso tienden a ser adultos obesos; por tanto, la prevención de la obesidad infantil constituye la prevención de la obesidad en la adultez. La obesidad en adultos es un factor de riesgo para el desarrollo de enfermedades crónicas: hipertensión, problemas cardíacos y diabetes, entre otras, importantes determinantes de la morbimortalidad temprana en la etapa adulta. Programas escolares que promuevan cambios en los hábitos alimentarios del niño y la familia son indispensables para establecer estilos de vida y alimentación saludable que perduren. ${ }^{32,33}$

El tipo de cobertura médica se asocia significativamente con la salud socioemocional: el $40 \%$ de los niños sin cobertura médica tienen salud socioemocional no óptima. Actualmente, el Hospital Zonal Bariloche no cuenta con servicio de atención psicológica infantil. Esto sugiere que los niños que más lo necesitan tienen mayor dificultad en obtenerla.

Los alcances de este estudio son acotados debido a limitaciones en la representatividad de la muestra. Además, solo uno de los médicos pediatras realizó encuestas en el Centro de Salud $y$, si bien la manera de abordar la entrevista fue cuidadosamente acordada entre los profesionales, esto podría representar una fuente de sesgo de la investigación. No obstante esto, el trabajo hace un importante aporte al conocimiento sobre desigualdades en la salud en la localidad, tema sobre el que no hay estudios previos. Los resultados alcanzados ponen de manifiesto la necesidad de atender a la salud infantil en la ciudad, especialmente la salud bucal, para evitar un mayor deterioro en los sectores más desfavorecidos.

Encontramos que cada característica sociodemográfica está independientemente asociada con alguna de las medidas de salud estudiadas. Además, detectamos que la posibilidad de resultados de salud desfavorables aumenta gradualmente con el número de factores de riesgo. Estos resultados coinciden con los de otros estudios sobre la importancia del número total de factores de riesgo sobre la salud infantil. 18,34 En este sentido, es esperable que los programas sociales y de salud que aborden multiplicidad de factores simultáneamente sean los que posean mayor potencial para influir sobre la salud de los niños.

Los problemas de salud repercuten sobre el rendimiento escolar del niño y, además, se asocian a una mayor posibilidad de embarazo adolescente, actividades delictivas, desempleo y depresión en la adultez. ${ }^{35}$ El conocimiento epidemiológico adquirido permitirá orientar la planificación de programas en el área de salud y desarrollo social tendientes a reducir las desigualdades en la salud en Bariloche y en otras localidades con características similares. Además, servirá de base para el desarrollo de proyectos de investigación relacionados con desigualdades en salud infantil.

\section{CONCLUSIONES}

Existen importantes brechas en la salud infantil de la muestra de escolares de Bariloche estudiada.

Múltiples factores de riesgo tienen efecto acumulado sobre el estado de salud general, socioemocional y bucal. La posibilidad de un estado de salud no óptimo aumenta 1,8 veces con cada factor de riesgo. El riesgo de problemas socioemocionales aumenta 1,4 veces con cada factor de riesgo, mientras que la posibilidad de caries o piezas faltantes se duplica.

Las prevalencias de sobrepeso y obesidad halladas son preocupantes: $27,3 \%$ y $8,7 \%$, respectivamente.

\section{REFERENCIAS}

1. Pan American Health Organization. Health statistics from the Americas, 1998. Washington DC; 1999.

2. Alleyne GA, Castillo-Salgado C, Schneider MC, Loyola E, et al. Overview of social inequalities in health in the region of the Americas, using various methodological approaches. Rev Panam Salud Publica 2002;12(6):388-97.

3. Lejarraga H, Pascucci M, Krupitzky S, Kelmansky D, et al. Psychomotor development in Argentinean children aged 0-5 years. Paediatr Perinat Epidemiol 2002;16(1):47-60.

4. Garibotti G, Comar H, Vasconi C, Giannini G, et al. Desarrollo psicomotor infantil y su relación con las características sociodemográficas y de estimulación familiar en niños de la ciudad de Bariloche, Argentina. Arch Argent Pediatr 2013;111(5):384-90.

5. Torralva T,Cugnasco I, Manso M,Sauton F, et al. Desarrollo mental y motor en los primeros años de vida: su relación con la estimulación ambiental y el nivel socio-económico. Arch Argent Pediatr 1999;97(5):306-16.

6. Chesta M, Lobo B, Agrelo F, Carmuenga E, et al. Evaluación antropométrica en niños de la ciudad de Córdoba, año 2000. Arch Argent Pediatr 2007;105(2):101-8.

7. Bejarano I, Dipierri J, Alfaro E, Quispe Y, et al. Evolución de la prevalencia de sobrepeso, obesidad y desnutrición en escolares de San Salvador de Jujuy. Arch Argent Pediatr 2005;103(2):101-9.

8. Kovalskys I, Bay L, Rausch Herscovici C, Berner E. Prevalencia de obesidad en una población de 10 a 19 años en la consulta pediátrica. Arch Argent Pediatr 2003;101(6):441-7.

9. Juiz de Trogliero C, Morasso MC. Obesidad y nivel socioeconómico en escolares y adolescentes de la ciudad de Salta. Arch Argent Pediatr 2002;100(5):360-6.

10. Poletti $\mathrm{OH}$, Barrios L. Obesidad e hipertensión arterial en escolares de la ciudad de Corrientes, Argentina. Arch Argent Pediatr 2007;105(4):293-8.

11. Llompart G, Marin GH, Silberman M, Merlo I, et al. Oral 
health in 6-year-old schoolchildren from Berisso, Argentina: falling far short of WHO goals. Med Oral Patol Cir Bucal 2010;15(1):e101-5.

12. Yankilevich ER, Cattoni ST, Cornejo LS, Battellino LJ. Distribución de la caries dental en niños preescolares en una región urbana, Argentina, 1992. Rev Saude Publica 1993;27(6):436-44.

13. Buchbinder M. Mortalidad infantil y desigualdad socioeconómica en la Argentina. Tendencia temporal. Arch Argent Pediatr 2008;106(3):212-8.

14. De Sarasqueta P. Mortalidad neonatal y posneonatal en recién nacidos de peso menor a $2500 \mathrm{~g}$ en la República Argentina (1990-1997). Arch Argent Pediatr 2001;99(1):59-61.

15. Sociedad Argentina de Pediatría. Guía para el pediatra. Invitemos a leer: Programa de promoción de la lectura. Buenos Aires; 2002.

16. National Research Council. Children's health, the Nation's wealth: assessing and improving child health. Washington DC: National Academies Press; 2004.

17. Blumberg SJ, Olson L, Frankel MR, Osborn L, et al. Design and operation of the National Survey of Children's Health, 2003. Vital Health Stat 1 2005;(43):1-131.

18. Larson K, Russ SA, Crall JJ, Halfon N. Influence of multiple social risks on children's health. Pediatrics 2008;121(2):337-44.

19. Brothers KB, Glascoe FP, Robertshaw NS. PEDS: developmental milestones- an accurate brief tool for surveillance and screening. Clin Pediatr (Phila) 2008;47(3):271-9.

20. Glascoe FP. Parents' evaluation of developmental status: how well do parents' concerns identify children with behavioral and emotional problems? Clin Pediatr (Phila) 2003;42(2):133-8.

21. Cole TJ, Bellizzi MC, Flegal KM, Dietz WH. Establishing a standard definition for child overweight and obesity worldwide: international survey. BMJ 2000;320(7244):1240-3.

22. Cole TJ, Flegal KM, Nicholls D, Jackson AA. Body mass index cut offs to define thinness in children and adolescents: international survey. BMJ 2007;335(7612):194.

23. Currie C, Molcho M, Boyce W, Holstein B, et al. Researching health inequalities in adolescents: The development of the
Health Behaviour in School-Aged Children (HBSC) family affluence scale. Soc Sci Med 2008;66(6):1429-36.

24. RDevelopment Core Team. R: a language and environment for statistical computig. Vienna: RFoundation for Statistical Computing; 2013.

25. Organización Mundial de la Salud. Determinantes sociales de la Salud. [Acceso: 14 de abril de 2015]. Disponible en: http://www.who.int/social_determinants/final_report/ key_concepts/es/

26. Braveman P. Health disparities and health equity: concepts and measurements. Annu Rev Public Health 2006;27:167-94.

27. Lurie N, Dubowitz T. Health disparities and access to health. JAMA 2007;297(10):1118-21.

28. Argentina. Ministerio de Salud de la Nación. Programa de Sanidad Escolar. [Acceso: 14 de abril de 2015]. Disponible en: http://www.msal.gov.ar/index.php/programas-yplanes / 229-programa-de-sanidad-escolar.

29. Ravera E,SanchezGA, Squassi AF, Bordoni N. Relationship between dental status and family, school and socioeconomic level. Acta Odontol Latinoam 2012;25(1):140-9.

30. Walker KK, Steinfort EL, Keyler MJ. Cues to action as motivators for children's brushing. Health Commun 2014:1-11.

31. Padilla IS. Prevalencia de sobrepeso-obesidad y factores asociados con valor predictivo-preventivo en escolares de 6 a 11 años de Río Gallegos, Santa Cruz, Argentina. Salud Colect 2011;7(3):377-88.

32. Flynn MA, McNeil DA, Maloff B, Mutasingwa D, et al. Reducing obesity and related chronic disease risk in children and youth: a synthesis of evidence with "best practice" recommendations. Obes Rev 2006;7(Suppl 1):7-66.

33. Avery A, Bostock L, McCullough F. A systematic review investigating interventions that can help reduce consumption of sugar-sweetened beverages in children leading to changes in body fatness. J Hum Nutr Diet 2015;28(Suppl 1):52-64.

34. Sameroff AJ.Environmental risk factors in infancy. Pediatrics 1998;102(5 Suppl E):1287-92.

35. Haskins R, Rouse C. Closing achievement gaps. Future Child. 2005. [Acceso: 14 de abril de 2015]. Disponible en: http: / futureofchildren.org/futureofchildren / publications/docs/15_01_PolicyBrief.pdf. 


\section{Anexo 1 \\ Estado de salud psicofísica infantil en relación con la situación socioeconómica: intercambio interdisciplinario, Centro Regional Universitario Bariloche - Centros de Salud de Bariloche}

Fecha:

Encuestador:

Institución:

Causa de la consulta: Control

Certificado médico

Otro (especifique):

Fecha de la última consulta (según historia clínica):

Datos de identificación

1. Edad: .......... años

2. Fecha de nacimiento:

No sabe

3. Peso al nacer:

Menos de 2,5 kg $\square \quad$ Más de 2,5 kg $\square \quad$ No sabe $\square$

4. ¿Fue prematuro?

Sí $\square$

No $\square$

No sabe

5. Sexo:

Femenino

Masculino

6. Concurre a un colegio:

Público

Privado

No concurre

No sabe

7. Grado escolar:

No sabe

8. ¿Cuántos hermanos (con los que comparte la madre) tiene el niño?

9. Persona que acompaña al niño:

Madre

Padre

10. Edad de la madre: años

No vive

Otro (especifique):

11. Edad de la madre al nacer el primer hijo:
Menos de 15 años
De 16 a 18 años
De 19 a 25 años
De 26 a 35 años
Más de 35 años
No sabe

12. Instrucción de la madre (marque el nivel máximo completado):
Ninguno
Primario
Secundario
Terciario
Universitario
No sabe
Otro:

13. Instrucción del padre (marque el nivel máximo completado):
Ninguno
Primario
Secundario.
Otro:
Universitario
No sabe

14. ¿El niño vive en la casa con la madre y el padre?

Sí

No

No sabe

15. ¿Algún miembro de la familia es de origen mapuche?

Sí

No

No sabe 


\section{Salud física y bucal}

1. Peso: $\mathrm{kg}$

Fecha de medición

2. Talla: $\mathrm{cm}$ Fecha de medición:

3. Presenta caries: Sí $\square$ No No sabe

4. Faltan piezas:
Sí
No
No sabe

5. Esquema de vacunación del Plan Nacional de Vacunación al día:
Sí $\square$
No
No sabe

6. Vacuna contra la varicela:
Sí
No
No sabe

7. Vacuna contra la hepatitis A:
Sí $\square$
No
No sabe

8. En general, ¿cómo describiría la salud del niño?

Excelente $\square \quad$ Muy buena $\square \quad$ Buena $\square \quad$ Regular $\square \quad$ Pobre $\square \quad$ No sabe

9. ¿Cómo describiría la condición de los dientes del niño?
Excelente
Muy buena
Buena
Regular
Pobre
No sabe

10. Considera que el niño come:
Poco
Regular
Adecuadamente
Mucho
Demasiado
No sabe $\square$

11. ¿Cuánto tiempo fue amamantado?
Nunca
Menos de 1 mes
Entre 1 mes y 3 meses
Entre 3 y 9 meses
Más de 9 meses
No sabe

12. Durante la última semana, ¿cuántos días el niño hizo ejercicio físico por más de 20 minutos que lo hizo transpirar o respirar agitadamente?
0 días $\square$
1-3 días $\square$
4-6 días $\square$
Todos los días
No sabe

13. Durante el último año, ¿aproximadamente, cuántos días de clase perdió el niño por estar enfermo o lastimado?
0 días
1-5 días
6-10 días
Más de 11 días
No sabe

14. Durante los últimos 12 meses, ¿el niño se lastimó y requirió atención médica?
Sí
No $\square$
No sabe

15. Durante los últimos 12 meses, ¿el niño estuvo internado alguna vez?
Sí
No
No sabe

16. Causas de las internaciones ocurridas durante los últimos 12 meses (marque todo lo que corresponda):

Patología respiratoria

Trauma o lesiones

Patología quirúrgica

Infecciones odontológicas

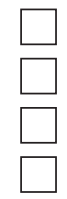

17. Durante los últimos 12 meses, ¿alguna vez el niño sufrió quemaduras?
Sí $\square$
No
No sabe 


\section{Salud emocional y mental}

1. Respecto de los siguientes temas, diga si usted está preocupado o no.

Cómo se comporta el niño

Preocupado

No preocupado

Cómo le va en la escuela

Cómo se relaciona con otros niños

Cómo lo tratan los compañeros

Cómo trata a los maestros y otras personas mayores

Su relación con la madre

Su relación con el padre

Cómo entiende el niño las consignas que se le dan

Depresión o ansiedad del niño

Violencia en la casa, la escuela o el barrio

Uso de drogas

Uso de alcohol

Desórdenes alimenticios del niño

Cómo resuelve el niño situaciones de conflicto

Cómo habla el niño

\section{Cobertura médica}

1. ¿Qué cobertura médica tiene?
Prepaga
Obra social
Ninguna
No sabe

2. Durante los últimos 12 meses, ¿hubo algún momento durante el cual no tuvo cobertura médica?
Sí
No
No sabe

3. La cobertura médica, ¿le cubre visitas de control al dentista y arreglos?
Sí $\square$
No
No sabe

4. Usualmente, cuando pide un turno (no de urgencia) para el médico del niño, se lo dan para: El mismo día o el día siguiente Entre 6 y 15 días después

Entre 3 y 5 días después Más de 15 días después

\section{Medicina preventiva y medicinas alternativas}

1. Durante los últimos 12 meses, ¿alguna vez llevó al niño al médico para un control general?
Sí $\square$
No
No sabe

2. Durante los últimos 12 meses, ¿alguna vez llevó al niño al dentista para un control?
Sí $\square$
No
No sabe

3. Durante los últimos 12 meses, ¿alguna vez tuvo que llevar al niño al dentista por arreglos?
Sí $\square$
No
No sabe

4. Durante los últimos 12 meses, ¿cuántas veces tuvo que llevar al niño al médico sin turno?
0
1
2 o más
No sabe

5. ¿El niño tiene un médico que lo conoce bien y está al tanto de su historia clínica?
Sí $\square$
No
No sabe

6. Durante los últimos 12 meses, ¿se ha consultado por el niño o por alguno de sus hermanos o padres algún tipo de medicina alternativa?
Ninguna
Homeopatía
Curandero
Otros (especifique): 
7. ¿Utiliza yuyos medicinales para tratar al niño?
Sí
No
No sabe

\section{Actividades escolares y recreativas}

1. Durante los últimos 12 meses, ¿el niño realizó actividades deportivas relativamente organizadas o tomó clases de algún deporte, fuera de lo requerido por el colegio?
Sí $\square$
No
No sabe

2. Durante los últimos 12 meses, ¿el niño participó de alguna otra actividad o evento organizado?
Sí $\square$
No
No sabe

3. En un día de clase, ¿aproximadamente, cuánto tiempo el niño lee o le leen por placer fuera del ámbito escolar?
Entre 30 minutos y una hora $\square$
Menos de 15 minutos $\square$
Más de una hora
Entre 15 y 30 minutos $\square$
No sabe

\section{Salud y actividades familiares}

1. Usted diría que, en general, la salud de la madre es:
Excelente
Muy buena
Buena $\square$
Regular $\square$
Pobre $\square$
No sabe

2. ¿La madre dialoga con sus hijos?
Todos los días
A veces $\square$

Nunca

No sabe

3. ¿La madre les pone límites a sus hijos?
Siempre $\square$
A veces
Nunca
No sabe

4. ¿Alguien en la casa fuma?

Sí

No

No sabe

5. ¿Alguien en la casa tiene problemas con el alcohol?
Sí $\square$
No
No sabe

6. Durante un día laborable, ¿aproximadamente, cuánto tiempo pasa la madre con el niño? Menos de $2 \mathrm{~h}$
Entre 2 y $4 \mathrm{~h}$
Entre 4 y $8 \mathrm{~h}$
Más de $8 \mathrm{~h}$
No sabe

7. ¿Alguien en la casa tiene auto?
Sí $\square$
No
No sabe

8. ¿Con cuántas personas comparte el niño el dormitorio?
Ninguna
1
2
3 o más
No sabe

9. Durante los últimos 12 meses, ¿cuántas veces el niño salió de vacaciones fuera de Bariloche por un período de más de 3 días?
Ninguna $\square$
$1 \square$
2
3 o más
No sabe

10. ¿Cuántas computadoras hay en la casa?
Ninguna $\square$
$1 \square$
2 o más $\square$
No sabe $\square$

11. En los últimos 12 meses, ¿alguien en la casa recibió algún tipo de subsidio o prestación alimentaria? (Por ejemplo: Subsidio por desempleo, Habitar en Familia, Comer en familia, PESA).
Sí $\square$
No
No sabe

12. Durante la última semana, ¿usted o algún familiar llevó al niño a pasear (plaza, casa de amigos o familiares, cerro, lago, restaurante, etc.)?
No $\square$
1-3 veces
4-6 veces
7 o más veces
No sabe

13. La semana pasada, ¿cuántos días el niño leyó solo o usted o algún familiar le leyeron un cuento o parte de una novela?
Ninguno $\square \quad$ 1-3 días
4-6 días
Todos los día $\square$
No sabe 
14. La semana pasada, ¿cuántos días todos los miembros de la familia comieron una comida juntos?

Ninguno $\square \quad$ 1-3 días $\square \quad$ 4- 6 días $\square \quad$ Todos los días $\square \quad$ No sabe $\square$

15. El último día que el niño fue al colegio, ¿desayunó antes de ir?
Sí
No
No sabe

16. En un día de clase, ¿aproximadamente, cuántas horas el niño mira televisión, videos o juega a juegos de computadora?

Ninguna $\square \quad$ Menos de una $\square \quad$ 1- 3 horas $\square \quad 4$ o más horas $\square \quad$ No sabe

17. La semana pasada, ¿algún día el niño estuvo solo en la casa, aun por un período corto?
Sí
No
No sabe

18. ¿Cuántas horas trabaja la madre fuera de la casa?
Ninguna
Menos de 4
4- 8 horas
8 o más horas $\square$
No sabe

19. Durante los últimos 12 meses, ¿alguien de la familia tuvo que dejar su trabajo o hacer algún cambio importante de trabajo para cuidar del niño?
Sí $\square$
No
No sabe

20. ¿Cuán seguido usted siente que el niño está seguro en el barrio?
Nunca
A veces
Usualmente
Siempre
No sabe

21. ¿Cuán seguido usted siente que el niño está seguro en la escuela?
Nunca
A veces
Usualmente
Siempre
No sabe 